\title{
Mass Spectrometry of Polymers: Polypropylene Glycol
}

\author{
Gregory M. Neumann, Peter G. Cullis, and Peter J. Derrick \\ La Trobe University, Physical Chemistry Department, Bundoora, Victoria 3083, Australia
}

Z. Naturforsch. 35a, 1090-1097 (1980); received July 30, 1980

Dedicated to Professor H.D.Beckey on the occasion of his 60th. birthday

\begin{abstract}
Mass spectra have been measured, using the field desorption technique, for polypropylene glycols with nominal mass-average relative molecular masses of $1000,2000,3000$ and 4000 . Ions of up to $m / z 7400$ have been observed. Major peaks in the spectra correspond to $(\mathrm{M}+\mathrm{H})^{+}$ions. $(\mathrm{M}+\mathrm{Na})^{+}$ions are observed if sodium iodide is added to the sample. There are strong peaks corresponding to fragmentation of the polymer chains close to their termini. It is suggested that these fragmentations are induced by the strong electric fields at the emitter.
\end{abstract}

\section{Introduction}

Mass spectrometry is, almost by definition, a method for measuring molecular masses precisely, and molecular mass is perhaps the most important property of a polymer. Yet, the use of mass spectrometry in polymer chemistry has for the most part been restricted to analysis of volatile products from degradation of polymers [1]. That mass spectrometry has not become a standard method for determining the molecular masses of polymers is due to the difficulties created by the low volatility and high masses of these compounds. Recently there have been some preliminary results, using specialized ionization techniques, which suggest that mass spectrometry may soon make a much more significant contribution to polymer chemistry. Using the field desorption technique [2] and a thin wire coated with silicon microneedles as the emitter, Matsuo et al. [3] have shown that gaseous pseudomolecular ions of up to $\mathrm{m} / \mathrm{z} 11000$ can be formed from polystyrene. Our interest in the mass spectrometry of synthetic polymers was stimulated by the fact that polyvinyl alcohol ions could be field desorbed from sharp edge emitters $[4,5]$. The purpose of this paper is to present the results of a detailed investigation of the field desorption of polypropylene glycols of nominal mass-average relative molecular masses 1000,2000 , 3000 and 4000 (from here on, the samples will be referred to as mass-1000, mass-2000, mass-3000 and mass-4000 respectively). These results show how, in addition to the determination of molecular

Reprint requests to Dr. P. J. Derrick, Physical Chemistry Department, La Trobe University, Bundoora, Victoria 3083, Australien. mass distributions, field desorption mass spectrometry can elucidate both the molecular structures of polymers and the chemistry of macromolecular ions.

The szecialized techniques used with non-volatile compounds generally rely on forming and desorbing ions from a solid probe, thereby circumventing the need for volatilization of the neutral prior to ionization $[6,7]$. In field desorption [7], strong electric fields are used for this purpose, although it is usually also necessary to gently heat the sample. The mechanism of field desorption, which is quite simple in the case of atoms [8], is more complex in the case of organic compounds [2]. Nevertheless, the field desorption approach has been shown to possess an efficacy for the production of molecular ions from thermally labile, non-volatile compounds [9], which is far beyond that of any other ionization method apart from californium-252 plasma desorption [6].

An obstacle to be surmounted in applying mass spectrometry to polymers is the magnitude of the magnetic field strength needed to deflect and analyse the massive molecular ions. Consider ions of $\mathrm{m} / \mathrm{z}$ 10000 , and a magnetic sector of radius $300 \mathrm{~mm}$. If their translational energy were $8000 \mathrm{eV}$, a magnetic induction of over $4 \mathrm{~T}$ would be required to transmit and focus such ions. Fields of this magnitude have not been realised in mass spectrometry experiments. Extending the mass range by lowering the ion energy leads to reduced sensitivity, and this is particularly true in the case of field desorption. Here the strong field, necessary to achieve significant ionization, also acts to accelerate the ions to higher energies, so that the beam, which is not collimated, must in fact be retarded if low energy ions are 
required for mass analysis $[2,10]$. Our response to this problem of analysing massive ions has been to employ a magnetic sector of large radius (nominally $780 \mathrm{~mm}$ ). This in turn influences the overall scale of the mass spectrometer.

Finally, massive ions of a given energy move at relatively slow velocities, leading to insensitivity of particle multiplier detectors [11]. Special techniques might therefore be desirable for the detection of massive ions [11], although in the work reported here a particle multiplier proved to be satisfactory for ions of up to $m / z 7400$.

\section{Experimental}

The emitters employed for field desorption were formed from tungsten wire ( $10 \mu \mathrm{m}$ diameter), using the standard activation technique [12]. Activation converts the wire substrate to tungsten carbide (WC) [13]. The magnitude of the emitter heating current was set manually, and feedback stabilised to better than $\pm 10 \mu \mathrm{A}$. The physical position of an emitter in the ion source was fixed, however the cathode slit could be adjusted sideways so as to maximise the mass-resolved signal [14]. All mass spectra were recorded with a potential of $8 \mathrm{kV}$ on the emitter and the cathode at earth potential.

The design and construction of the double-focussing mass spectrometer employed have been outlined elsewhere [14, 15] and will be discussed in detail in relation to performance in a future paper [16]. The magnetic sector radius is nominally $780 \mathrm{~mm}$ and the electric sector radius is $1000 \mathrm{~mm}$. The widths of the source and collector slits were fixed at $0.25 \mathrm{~mm}$ and $0.40 \mathrm{~mm}$ respectively, which gave a mass resolution of approximately 2000. The magnetic induction was sensed using magnetoresistor circuits.

Up to $m / z 1000$, mass calibration of the polypropylene glycol spectra was based on the field ionization mass spectra of perfluorokerosene and perfluorotributylamine. Up to $m / z 2500$, there were sufficient peaks in the mass spectra of the glycols to allow "counting" of peaks. Above $m / z 2500$, mass assignment rests on the reproducibility of the measured values of the magnetic induction. The uncertainty in mass assignments above $\mathrm{m} / z 2000$ is placed at \pm 0.001 , e. g. uncertainty in $\mathrm{m} / z 5000$ is \pm 5 . If, however, it is assumed that the type of ions formed above $m / z 2500$ with the mass-2000, 3000 and 4000 samples are the same as those formed with the mass-1000 sample, the mass assignments at all masses are reliable to better than \pm 0.5 of an $\mathrm{m} / z$ unit.

The polypropylene glycol samples were purchased from Aldrich. Gel permeation chromatography was used to test the purity. Samples were loaded onto the emitters either as the pure compounds or dissolved in a solvent (acetone, water or ethanol/water). In certain experiments, various amounts of mineral acid were added to sample solutions to encourage protonation.

\section{Results}

The polypropylene glycols could be field desorbed at room temperature. Passing a heating current of up to $15 \mathrm{~mA}$ through the emitter in general enhanced the ion emission (see below). The total emitted ion currents could be as high as $10 \mathrm{nA}$, and could persist for some hours. The usual concept of a "best anode temperature" [9] was not relevant to these experiments, since ion desorption occurred smoothly over a broad range of heating currents.

The field desorption mass spectra of the poly. propylene glycol samples are shown in Figures $1-3$. Regions of the mass-1000 spectrum are shown in greater detail in Figure 4. The large numbers of peaks in each spectrum can be arranged into a relatively small number of series of peaks, in which successive members are separated by $58 \mathrm{~m} / z$ units. $58 \mathrm{a} . \mathrm{m}$. u. is the repeat unit of polypropylene glycol. Indeed, the only peak in the spectra which does not appear to be part of some series is the $m / z 87$ peak, the origin of which is unclear. The alteration in peak intensity on moving along a series is generally gradual as is evident from Figures $1-3$. This allows the concept of an "intensity envelope" to be used to mean the distribution curve which would result from drawing a continuous line through the tops of all the peaks in any particular series. There appears to be 6 strong series, composed of peaks corresponding to the ions of the general formulae $(M+1)^{+}$, $(M-17)^{+},(M-18)^{+} .,(M-31)^{+},(M-103)^{+}$and $(M-104)^{+} . . M$ devotes the relative molecular masses of the polymer, which are given by the formula $M=n 58+18$ ( $n$ is an integer). All six series occur with each of the four samples of polypropylene glycol, however the $(M-103)^{+}$and $(M-104)^{+}$. series are very weak in the mass-3000 and mass4000 spectra and are not shown in Figure 3. 


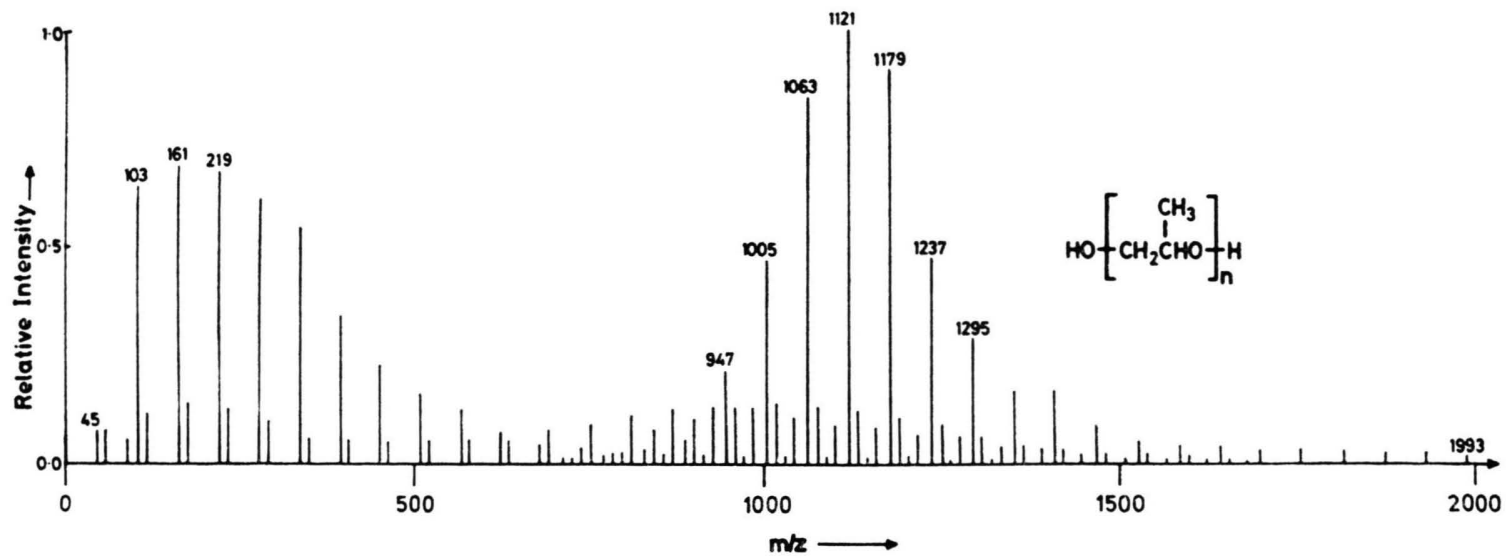

Fig. 1. The field desorption mass spectrum of the mass-1000 polypropylene glycol. Emitter heating current was $13.0 \mathrm{~mA}$. ${ }_{13} \mathrm{C}$ isotope peaks have been omitted.

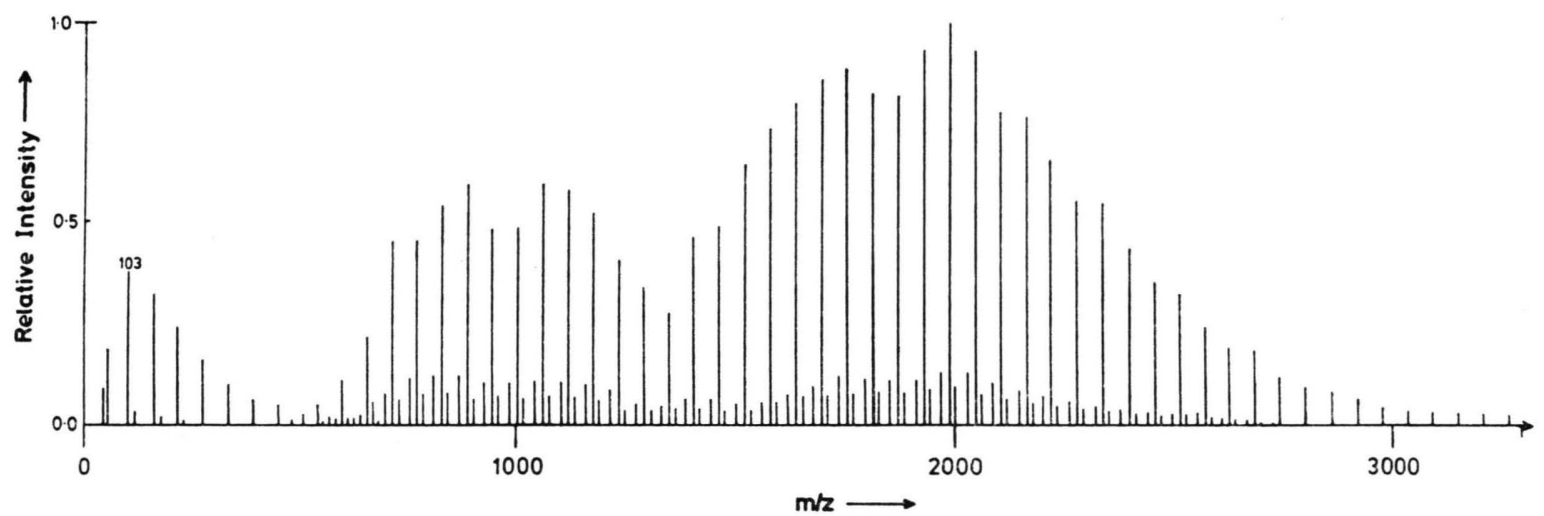

Fig. 2. The field desorption mass spectrum of the mass-2000 polypropylene glycol. Emitter heating current was $13.0 \mathrm{~mA}$. ${ }^{13} \mathrm{C}$ isotope peaks have been omitted.

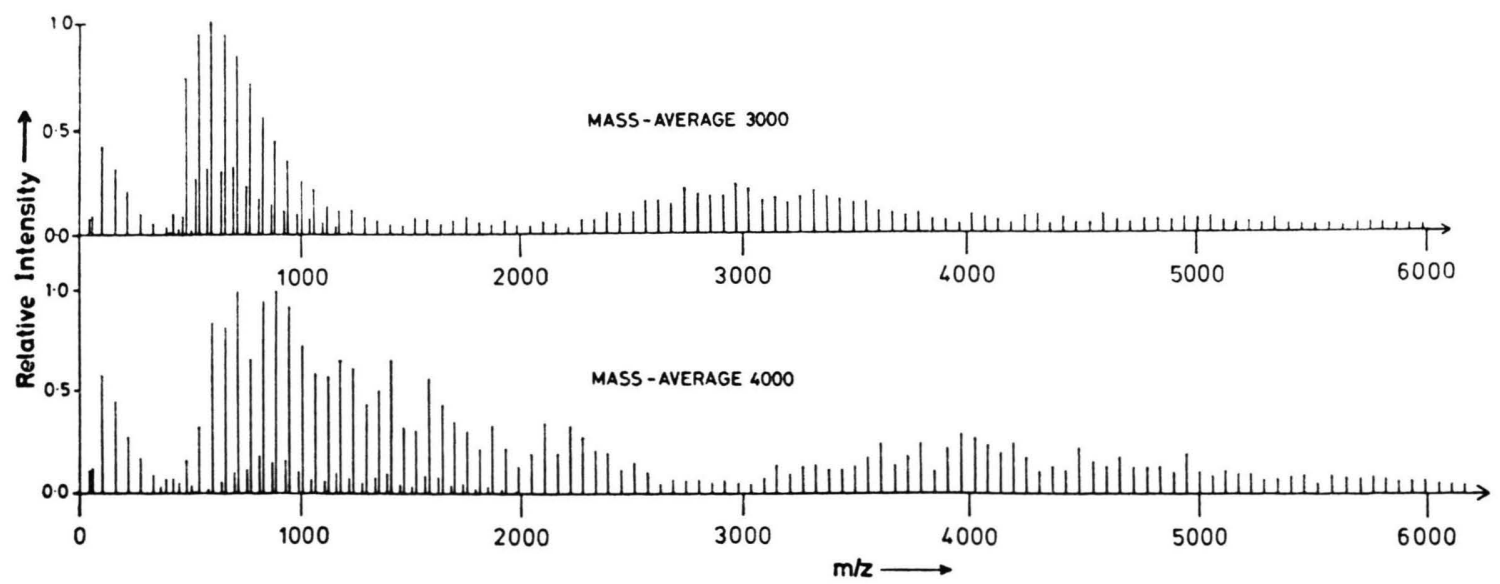

Fig. 3. The field desorption mass spectra of the mass-3000 and mass-4000 polypropylene glycol. Emitter heating current was $13.0 \mathrm{~mA}$ for mass-3000 and $15.0 \mathrm{~mA}$ for mass-4000. Only peaks in the $(\mathrm{M}+1)^{+},(\mathrm{M}-18)^{+}$and $(\mathrm{M}-31)^{+}$series are shown. ${ }^{13} \mathrm{C}$ isotope peaks have been omitted. 


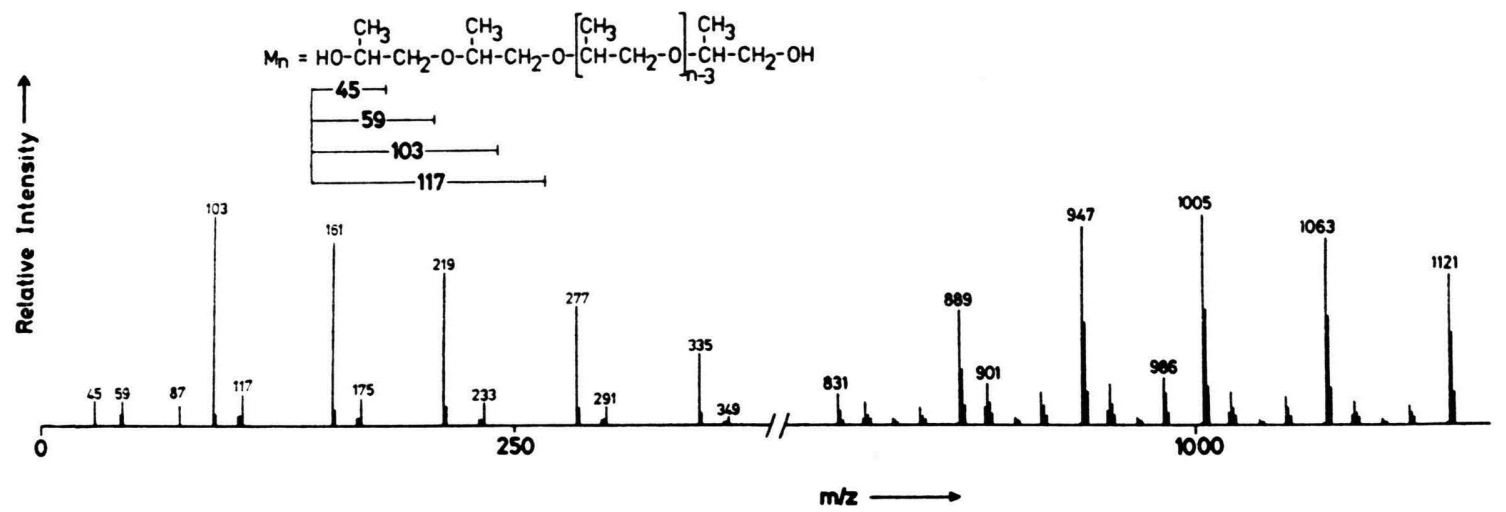

Fig. 4. Two regions of the field desorption mass spectrum of the mass-1000 polypropylene glycol. Emitter heating current was $12.0 \mathrm{~mA}$.

The $(M-103)^{+}$is designated as such rather than $(M-45)^{+}$, because it appears that this fragment is derived from the polymer molecule whose mass is 103 a.m.u. greater than that of the fragment ion. This conclusion is based on the fact that the intensity envelope for the $(M-103)^{+}$series is very similar to that of the $(M+1)^{+}$series, except that the former is shifted to lower masses by $104 \mathrm{~m} / z$ units. The intensity envelopes of the $(M-18)^{+}$and $(M-104)^{+}$. series are also similar to that of the $(M+1)^{+}$series. That of the $(M-18)+$ series is shifted to lower mass by $19 \mathrm{~m} / z$ units, compared to the $(M+1)^{+}$series, and that of $(M-104)^{+}$. by $105 \mathrm{~m} / z$ units. The intensity envelopes of the $(M-31)^{+}$series, which includes $\mathrm{m} / z 45, \mathrm{~m} / z \mathrm{103}$, $m / z 161, m / z 219, m / z 277$ and $m / z 335$, and the $(M-17)^{+}$series, which includes $m / z 59, m / z 117$, $m / z 175, m / z 233, m / z 291$ and $m / z 349$, are evidently very different from that of the $(M+1)^{+}$series
(Figures $1-3$ ). There is admittedly a degree of inconsistency in the naming of these last two series, in that $(M-31)^{+}$and $(M-17)^{+}$are not intended to signify that the fragment ions are derived from polymer molecules whose masses are 31 or $17 \mathrm{a} . \mathrm{m}$. u. greater than those of the fragments (see below).

The peak intensities in the mass spectra of all four samples were dependent upon heating current. Figure 5 shows mass spectra of the mass-1000 sample at a number of different heating currents. The room-temperature $(0 \mathrm{~mA})$ mass spectrum was run first. The heating current was then switched on, and a mass spectrum run at $10.0 \mathrm{~mA}$. The $11.5 \mathrm{~mA}$, $13.0 \mathrm{~mA}$ and $15.0 \mathrm{~mA}$ spectra were subsequently run in that order. It is evident from Fig. 5 that the higher heating currents favour the desorption of higher mass ions, and this was the case with all the samples. If the heating current was returned to a lower value after a mass spectrum had been run at

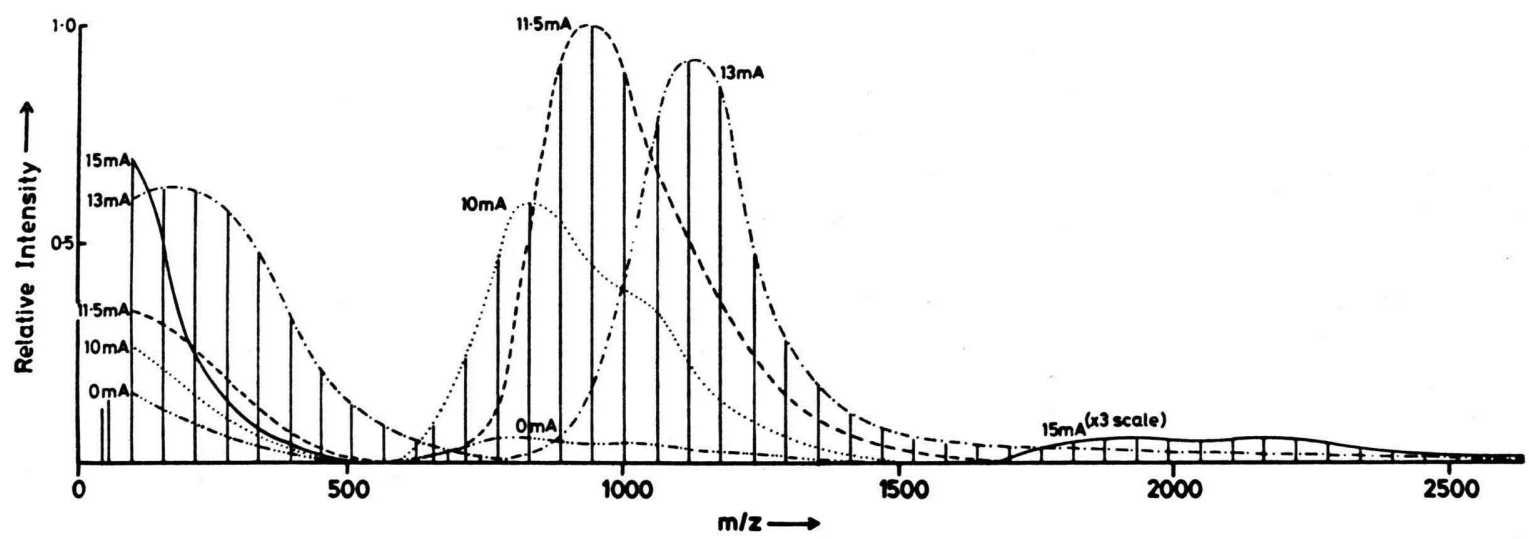

Fig. 5. Field desorption mass spectra of the mass-1000 polypropylene glycol at a number of different emitter heating currents. Only the intensity envelopes of the $(M+1)^{+}$and $(M-31)^{+}$series are shown. 


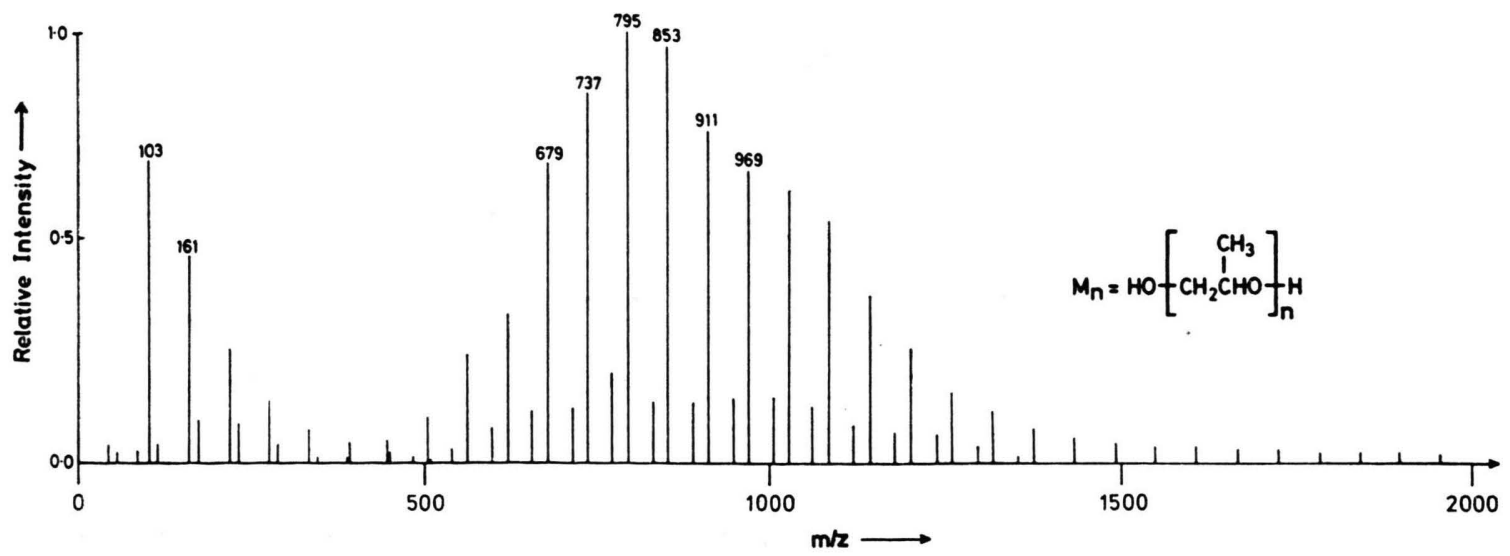

Fig. 6. Field desorption mass spectrum of mass-1000 polypropylene glycol to which aqueous sodium iodide has been added. Emitter heating current was $10.0 \mathrm{~mA}$. Only peaks in the $(\mathbf{M}+\mathrm{Na})^{+},(\mathbf{M}+1)^{+},(\mathrm{M}-31)^{+}$and $(\mathrm{M}-17)^{+}$series are shown. ${ }^{13} \mathrm{C}$ isotope peaks have been omitted.

a higher value, the mass spectrum characteristic of the higher temperature was still observed but at reduced intensity. Overall, the degree of fragmentation was found to be insensitive to emitter heating current with all four samples. There was a certain dependence of the relative intensities of the peaks in the $(M-17)^{+}$and $(M-31)^{+}$series upon heating current, which is shown in Figure 5. Otherwise, the only pronounced effect was that the intensities of the $(M-18)^{+}$. peaks fell on raising the heating current.

Matsuo et al.[3] have reported that the major peaks in the FD mass spectrum of polypropylene glycol (mass-1000) are due to $(M+\mathrm{Na})^{+}$ions. $(M+\mathrm{Na})^{+}$ ions were not observed in our experiments, unless the samples were deliberately contaminated with sodium. Figure 6 shows a mass spectrum of the mass-1000 sample to which aqueous sodium iodide (to a concentration of approximately $1 \mathrm{M}$ ) had been added. The major peaks at high masses correspond to cationized molecules $(M+\mathrm{Na})^{+}$; the $(M+1)^{+}$ peaks are still present. No sodium-cationized equivalents of the other series of peaks were observed. One effect of doping the samples with sodium iodide was that the rate of desorption became more sensitive to emitter heating current, and, in contrast to experiments on the polymer in the absence of salts, there was an optimum heating current, or "best anode temperature". The polymer has also been cationized using potassium and caesium.

\section{Discussion}

The $(M+1)^{+}$peaks are assigned to protonated molecules $(M+\mathrm{H})^{+}$, so that an obvious feature of the results is that these pseudomolecular ions are formed to the exclusion of molecular ions $(M)+$. That molecular ions are not formed is attributed to the magnitude of the field strengths [2] experienced by the polymers. The polymer, existing in a condensed phase on the emitter surface, is probably adsorbed in regions where field strengths are not sufficiently high to induce field ionization.

It is suggested that the pseudomolecular ions $(M+\mathrm{H})^{+}$are formed by proton transfer from one polymer molecule to another, and that this is induced by the electric field (of, perhaps, $10^{8} \mathrm{Vm}^{-1}$ ) [2]. Röllgen, Beckey and colleagues [17] and Block and colleagues [18] have found that proton transfer reactions can proceed at field strengths below the threshold for field ionization. Desorption would occur due to the force of the field on the protonated molecule overcoming the intermolecular forces holding the molecule at the surface. The enhanced desorption upon heating is attributed to disruption of the restraining intermolecular forces. The tendency for smaller molecules to desorb at lower heating currents would result from the smaller number of intermolecular bonds, as compared to a larger molecule. That the transferred proton originates from another polymer molecule can be inferred from the fact that $(M+\mathrm{H})^{+}$are formed in the absence of solvent.

A distinctive feature of the field desorption of polypropylene glycol is that desorption occurs smoothly and in a controllable fashion over a wide range of emitter heating currents $(0$ to $15 \mathrm{~mA})$. This relative insensitivity to heating current is 
perhaps due to the fact that the polypropylene glycol is a liquid even at room temperature. The existence of "best anode temperature" with other compounds may reflect phase changes in the sample.

The distribution of $(M+\mathrm{H})^{+}$peaks in the mass spectrum of the mass-average 1000 sample (Fig. 1) is considered to be related to the molecular mass distribution of the polymer. It is clear, however, that in general, the intensity distribution of the $(M+\mathrm{H})^{+}$ peaks at any particular heating current is not an exact measure of the mass distribution, since the intensity distribution is not constant but varies with changes in emitter heating current (Figure 5). To obtain the exact molecular mass distribution it would be necessary to determine correction factors to be applied to the $(M+\mathrm{H})^{+}$intensities. These factors would allow for dependence of ion production upon molecular mass, for possible loss of neutral polymer molecules from the emitter and for the usual mass discrimination possible with any mass spectrometer (in this instance, multipler response may be the major concern). The possibility of polymerisation and depolymerisation occurring on the emitter must also be kept in mind, although there is no evidence from the results presented here of such processes occurring with polypropylene glycol.

The distribution of $(M+\mathrm{H})^{+}$peaks in the mass 2000 spectrum (Fig. 3) is again considered to be related to the molecular mass distribution, without necessarily being an exact measure. The salient features of the mass-3000 and 4000 spectra (Fig. 4) are the intense $(M+\mathrm{H})^{+}$peaks at low masses (ca. $m / z 1000)$. There was a definite low-mass tail under gel permeation chromatography. The explanation is that the heating currents employed for the mass- 3000 and mass -4000 samples were such as to favour desorption of lower mass ions $(m / z<2000)$. Higher heating currents brought up the relative intensities of the peaks from $m / z 2000-6000$, but also decreased the desorption times of lower mass ions.

The fragment ions in the polypropylene glycol mass spectra bear a direct relationship to the neutral molecular structure. It is also clear that fragmentation occurs predominantly in bonds close to the ends of the chains. This fact strongly suggests that the electric field plays a significant role in inducing fragmentation, since the field will tend to locate the positive charge at (and hence weaken) bonds at the emitter-distant end of the molecular chains [2]. That thermal decomposition is not responsible for the fragments in the polypropylene glycol mass spectra is also indicated by the general tendency for fragmentation to either be insensitive to temperature or even to be reduced on raising the heating current.

The $(M-31)^{+}$series of fragment ions $(m / z 45$, $m / z 103, m / z 161$ etc.) are attributed to field-induced reactions. Possibly a hydrogen transfer is concerted with $\mathrm{C}-\mathrm{C}$ bond scission (Scheme (1)).

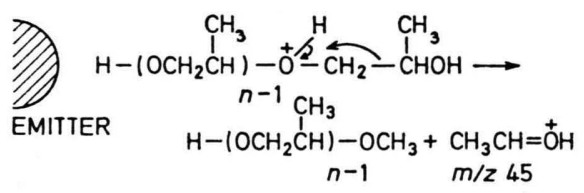

AND

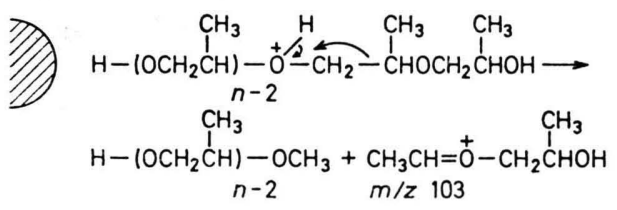

AND SO ON

It is supposed that, upon formation in a condensed phase, the $(M+\mathrm{H})^{+}$ions begin to move away from the emitter under the control of the electric field. The molecule will tend to align its chain length with the field lines so as to maximise the polarisation and the positive charge will be located at the emitterdistant end of the molecule [2]. Consider an electric field of $10^{9} \mathrm{Vm}^{-1}$. The $\mathrm{m} / z 1005$ ion, for example, would travel $5 \mathrm{~nm}$ in $10 \mathrm{ps}$, and the $m / z 5006$ about $1 \mathrm{~nm}$ in $10 \mathrm{ps}$. These distances compare to estimated extended chain lengths of $6 \mathrm{~nm}$ and $31 \mathrm{~nm}$ respectively. After $10 \mathrm{ps,}$, therefore, the massive ions will still be within a few $\mathrm{nm}$ of the ionization region, but aligned to some extent with the field and with the positive charge located towards the emitter-distant end of the molecule.

The $(M+17)^{+}$series of fragment ions $(m / z 59$, $m / z 117, m / z 175$ and so on) can be explained in terms of straight-forward field dissociation (II). Field dissociation at the other end of the molecule

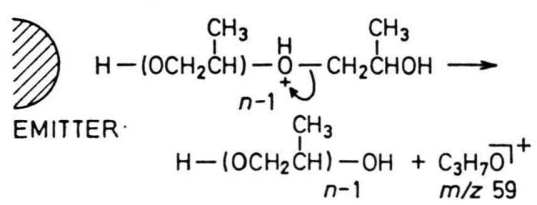

can also lead to these ions (III). Both of the proposed reactions ((II) and (III)) would be 
$\geqslant$ EMITTER

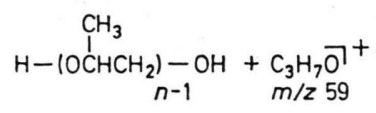

favoured by the fact that a stable molecule (polypropylene glycol) is being formed.

If the polypropylene glycol formed in the proposed reactions (Scheme II and III) were subsequently ionized, $(M+\mathrm{H})^{+}$ions would presumbly be produced and contribute to these peaks in the spectra. It appears, however, that the neutral fragments from the various field-induced reactions are not ionized, otherwise the neutrals from the reaction (I) should be visible in the mass spectrum. These neutrals have masses $(M-102)$, and if ionized should occur as $(M-101)^{+}$in the mass spectrum. There are no ions at these masses. The neutral fragments presumably escape from the ionization region.

The $(M-103)^{+}$-series of fragment ions might be explained on the basis of the mechanism (IV). That the intensities of these ions are always weak can be explained on the grounds that the charge resides on the fragment closest to the emitter [2]. As before, the reason for the rupture of this

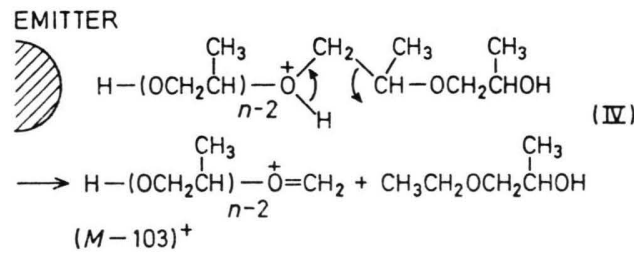

particular C-C bond (Scheme IV), rather than any of the other $\mathrm{C}-\mathrm{C}$ bonds in the chain, is seen as the positive charge tending to be localized in this region due to the influence of the field.

The $(M-18)^{+}$and $(M-104)^{+}$series of frag. ment peaks differ from the other fragments discussed in that they are odd-electron species. The $(M-104)^{+}$is a minor peak in all the spectra, however $(M-18)+$ is a major fragment ion with the mass-3000 and mass- 4000 spectra. The formation of the odd-electron ion $(M-18)+$ from the even-electron $(M+\mathrm{H})^{+}$would not have been ex-

[1] (a) D. O. Hummel, in "Polymer Spectroscopy", ed. D. O. Hummel, Verlag Chemie, Berlin 1974. (b) C. David in "Degradation of Polymers", Vol. 14 of "Comprehensive Chemical Kinetics", eds. C. H. Bamford and C. F. H. Tipper, Elsevier, Amsterdam 1975. pected to be a favourable process. A thermal decomposition does not seem to be involved, since the $(M-18)+$ intensities fall on raising the heating current.

\section{Conclusion}

Using the technique of field desorption gaseous pseudomolecular ions $(M+\mathrm{H})^{+}$can be formed from polypropylene glycols of nominal mass-average relative molecular masses $1000,2000,3000$ and 4000. These are polymers of relatively low molecular masses, but there is the possibility that the technique will be equally successful with compounds of higher masses. It is pointed out that there is no noticeable fall in ionization efficiency on moving from $m / z 3000$ up to $m / z 7000$.

The determination of molecular mass distributions of polymers by mass spectrometry appears to be feasible, at least for low molecular masses. What is required are mass standards, which can be used to obtain the correction factors for the pseudomolecular ion intensities.

The long-chain pseudomolecular ions undergo field-induced reactions to an extent which would be quite unusual for smaller molecular ions. The fieldinduced fragmentation provides structural information about the groups at the ends of the polymer chains.

Finally, it is emphasised that the ion emission can be intense (order of $10 \mathrm{nA}$ ) and stable over lengthy periods of time (hours), opening up possibilities for other experiments such as collisional decomposition [19] or laser spectroscopy [20] of the beam of massive ions.

\section{Acknowledgements}

We are pleased to acknowledge the financial support of the Australian Research Grants Committee under Grant No. C76/15797. We are delighted to be able to express our gratitude to Mr. John Chippindall for his invaluable contribution to this research project. We are indebted to Dr. Ken Ghiggino of Melbourne University for gel permeation chromatography results and for valuable discussion of the mass spectrometry results.

[2] H. D. Beckey, Principles of Field Ionization and Field Desorption Mass Spectrometry, Pergamon Press, Oxford 1977.

[3] T. Matsuo, H. Matsuda, and I. Katakuse, Anal. Chem. 51, 1329 (1979). 
[4] M. G. Darcy, M. Sc. Thesis, La Trobe University 1979.

[5] See also R. D. Lattimer, D. J. Harman, and K. R. Welch, Anal. Chem. 51, 1293 (1979).

[6] R. D. Macfarlane, C. J. McNeal, and J. E. Hunt, Adv. Mass Spectrom., 8 (1980).

[7] H. D. Beckey, Int. J. Mass Spectrom. Ion Phys. 2, 500 (1969).

[8] E. W. Müller, Phys. Rev. 102, 618 (1956).

[9] H. D. Beckey and H.-R. Schulten, Agnew, Chem. Int. Ed. 14, 403 (1975).

[10] A. J. B. Robertson and B. W. Viney, J. Chem. Soc. A 88, 1843 (1966).

[11] R. J. Beuhler and L. Friedman, Int. J. Mass Spectrom. Ion Phys., 23, 81 (1977).

[12] H. D. Beckey, E. Hilt, and H.-R. Schulten, J. Phys. E. Sci. Instrum. 6, 1043 (1973).

[13] G. M. Neumann, D. E. Rogers, P. J. Derrick, and P. J. K. Paterson, J. Phys., D: Appl. Phys. 13, 485 (1980).
[14] P. G. Cullis, G. M. Neumann, D. E. Rogers, and P. J Derrick, Adv. Mass Spectrom., 8 (1980).

[15] M. G. Darcy, D. E. Rogers, and P. .J. Derrick, Int. J. Mass Spectrom. Ion Phys., 27, 335 (1978).

[16] A. G. Craig, P. G. Cullis, K. F. Donchi, G. M. Neumann, D. E. Rogers, and P. J. Derrick, to be published.

[17] (a) F. W. Röllgen, H. J. Heinen, and U. Giessmann, Naturwiss. 64, 222 (1977). (b) F. W. Röllgen and H. D. Beckey, Z. Naturforsch. 29 A, 230 (1975). (c) F. W. Röllgen and H.-R. Schulten, Z. Naturforsch. 30 A, 1685 (1975).

[18] N. Ernst, G. Bozdech, and J. H. Block, Int. J. Mass Spectrom. Ion Phys., 28, 33 (1978).

[19] F. W. McLafferty, Accounts Chem. Res. 13, 33 (1980).

[20] A. Carrington, Proc. Roy. Soc. London 367 A, 443 (1979). 